\title{
Solar Neutron and Gamma-ray Spectroscopy Mission: SONGS
}

\section{Kazutaka Yamaoka, ${ }^{a, *}$ Hiroyasu Tajima, ${ }^{a}$ Daiki Nobashi, ${ }^{a}$ Masaki Usami, ${ }^{a}$ Kikuko Miyata, ${ }^{b}$ Takaya Inamori, ${ }^{c}$ Ji Hyun Park, ${ }^{c}$ Kazuya Ito, ${ }^{d}$ Koji Matsushita, ${ }^{d}$ Toyoki Watabe, ${ }^{d}$ Kazuhiro Nakazawa, ${ }^{e}$ Satoshi Masuda, ${ }^{a}$ Hiromitsu Takahashi ${ }^{f}$ and Kyoko Watanabe $^{g}$}

${ }^{a}$ Institute for Space-Earth Environmental Research (ISEE), Nagoya University, Nagoya, Japan

${ }^{b}$ Department of Vehicle and Mechanical Engineering, Meijo University, Nagoya, Japan

${ }^{c}$ Department of Aerospace Engineering, Graduate School of Engineering, Nagoya University, Nagoya, Japan

${ }^{d}$ Technical Center, Nagoya University, Furo-cho, Chikusa-ku, Nagoya, Japan

${ }^{e}$ Kobayashi-Maskawa Institute for the Origin of Particles and the Universe (KMI), Nagoya University, Nagoya, Japan

${ }^{f}$ Department of Physical Science, Graduate School of Science, Hiroshima University, Higashi-Hiroshima, Japan

${ }^{g}$ Department of Earth and Ocean Sciences, National Defense Academy of Japan, Yokosuka, Japan E-mail: yamaoka@isee.nagoya-u.ac.jp

Fast neutrons generated by the interaction between ions and the solar atmosphere are important observation problems to clarify the ion acceleration mechanism in the Sun, but so far neutrons have been detected from only $12 \mathrm{X}$-class solar flares in the highland on the ground due to the influence of atmospheric absorption. As for observations in space, SEDA-AP at the International Space Station continued to operate until 2018 and succeeded in neutron detection from 52 solar flares, but there are currently no dedicated space missions. In order to overcome this situation, we have been designing and developing $3 \mathrm{U}$ CubeSat and novel neutron/gamma-ray sensors since 2018 with the aim of performing satellite observations from outer space. The sensor consists of the multi-layered plastic scintillator bars readout with Si PM, which is a semiconductor photo-sensor, and detects fast neutrons from the tracks of ejected protons by elastic scattering. Furthermore, by placing GAGG scintillator arrays at the bottom, it is designed to be sensitive to gamma-rays based on the principle of the Compton camera. In this presentation, we will report on the scientific motivation and the development status of CubeSat and neutron/gamma-ray sensors.

$37^{\text {th }}$ International Cosmic Ray Conference (ICRC 2021)

July 12th-23rd, 2021

Online - Berlin, Germany

\footnotetext{
*Presenter
} 


\section{Introduction}

It is known that the Sun is one of the origin of cosmic-rays up to $10 \mathrm{GeV}$ (solar cosmicrays). Strong solar flares and associated coronal mass ejections (CME) accelerates large amount of charged particles such as ions and electrons, and they sometimes affect human life on the Earth due to destruction of satellites and power shortage. Reconnection of magnetic loops [1] is one of the most important physical processes that release the magnetic energy and accelerate particles, but it is still unknown how, when and where particles are accelerated in the Sun. Most studies on particle acceleration mechanism have been performed with electro-magnetic waves such as hard X-rays and microwaves which is related to electron acceleration. Ion acceleration mechanism is not well understood in compared to electron acceleration, and can be revealed only through nuclei-excited gamma-ray lines, e.g. $2.2 \mathrm{MeV}$ line due to neutron capture of hydrogen atoms, in the $\mathrm{MeV}$ region, $\pi_{0}$ decay in $\mathrm{GeV}$ gamma-rays, and neutrons produced by interaction with solar atmosphere. $\mathrm{GeV}$ gamma-rays were observed and delayed emission probably due to shock acceleration in the CME was confirmed by Fermi satellite since 2008 [2].

Solar neutrons have been mainly observed from highlands by neutron monitors and telescopes since 1980's [3], and there is only 12 detection [4] in associated with X-class solar flares over 40 years since its discovery in 1982 [5]. This is probably because neutron flux is significantly attenuated by the Earth's atmosphere and detection sensitivity is not so good due to high background. Spacebased observation has been also made by SEDA-AP on the International Space Station (ISS) [6][7] and MESSENGER in Mercury orbit [8]. Although the ISS detector is relatively small $\left(100 \mathrm{~cm}^{2}\right)$ in compared with ground-based detector with a large area of a few $10 \mathrm{~m}^{2}$, it has detected neutrons from more than 40 solar flares including M-class flares. MESSENGER also detected neutron events from a few solar flares. However neither mission is active now, and no space mission dedicated to solar neutrons is planned in near future.

To overcome this situation where there is no dedicated mission in space, we have designed and developed solar neutron sensors for microsatellite. The satellite we already launched is the ChubuSat-2 satellite with a $50 \mathrm{~kg}$ weight on February 17, 2016, but it has not been successfully operated in orbit [9]. To recover the Chubusat-2 mission, we have a plan to launch a $3 \mathrm{U}$ CubeSat dedicated for solar neutron observations around 2024 at the next solar maximum [10]. This project is so-called SOlar Neutron and Gamma-ray Spectroscopy mission (SONGS), and will be the first specific satellite to observe solar neutrons. In this paper, we describe overview of satellite and mission instrument, and present several results from blead board model (BBM).

\section{SONGS project}

\subsection{Overview}

The SONGS is a $3 \mathrm{U}$ CubeSat with a size of $10 \mathrm{~cm} \times 10 \mathrm{~cm} \times 34 \mathrm{~cm}$ amd a weight of $4 \mathrm{~kg}$. This project is based on concept design of the 1st Nagoya University CubeSat (NuCube-I), MAGNARO, lead by engineering school of Nagoya University, which will be launched by the Epsilon rocket next year. The MAGNARO is formation flight mission between $1 \mathrm{U}$ and $2 \mathrm{U}$ CubeSats separated in orbit without any thrusters. The SONGS utilizes the bus system of the $2 \mathrm{U}$ satellite and carries a mission radiation sensor which is sensitive to both neutrons and gamma-rays and its support instruments 
mentioned later. The main scientific goal of the SONGS is to reveal ion acceleration mechanisms by measuring precise neutron energy spectrum and occurrence timing of the neutron emission for some solar flares, and the second goal is to inform occurrence of large solar flares to all over the world as soon as possible from point of view of space weather by detecting soft gamma-rays (100 $\mathrm{keV}-2 \mathrm{MeV}$ ) which proceeds any other emission.

The mission instrument can track cosmic-ray interaction in the three dimensions, and requires many sensors and signal processing channels (more than 700). This means that they require large power consumption ( $4 \mathrm{~W}$ ) and large data size for downlink (15 Mbyte per day). To satisfy this criteria, we need deployable solar paddle to keep the sensor active and S-band communication system to ensure a large bandpath in addition to the MAGNARO bus system. On the other hand, attitude control stability is not so strict within 10 degrees. The mission requirement to the satellite bus system is summarized in Table 1.

Table 1: Mission requirement.

\begin{tabular}{ccc}
\hline \hline Items & Value & Requirement to the bus \\
\hline Weight & $\sim 2 \mathrm{~kg}$ & \\
Power & $\sim 4 \mathrm{~W}$ & Deployable solar paddles \\
Operation/Storage Temperature & $-20 \sim 20 /-20 \sim 60$ deg. C & Radiator etc. \\
Attitude & Sun pointing (within 10 deg.) & \\
& Determination accuracy: $1-2$ deg. & Earth horizon sensor \\
Data size (Mbyte/day) & $\sim 15$ (maximum), 1 (compressed) & S-band antenna \& transceiver \\
Mission Life & More than one year & higher altitude than the ISS orbit \\
Absolute Timing Accuracy & 1 sec (1 msec for gamma-rays) & PPS signals in GPS \\
Others & Protection of solar optical-UV light & \\
\hline
\end{tabular}

\section{Solar Neutron and Gamma-ray Spectrometer}

The design of the SONGS mission instrument is based on FIBer detector of the SEDA-AP on the ISS which utilizes 512 plastic scintillators, readout by multi-anode photo-multiplier tubes (PMTs). To make the sensor applicable for microsatellite, we replaced multi-anode PMTs with more compact Multi-Pixel Photo Counters (mPPCs). We have further added gamma-ray detection function by putting $\mathrm{GAGG}\left(\left(\mathrm{Gd}_{3} \mathrm{Al}_{2} \mathrm{Ga}_{3} \mathrm{O}_{12} ; \mathrm{Ce}\right) ; \mathrm{Ce}\right)$ array at the bottom of the sensor. The size and weight of the sensor would be $10 \mathrm{~cm} \times 10 \mathrm{~cm} \times 12 \mathrm{~cm}$ and $2 \mathrm{~kg}$ (see Table 2).

The neutron/gamma-ray sensor consists of three parts. One is plastic scintillator array (PSA) with 16 layers of 16 plastic scintillator bars with a size of $64 \mathrm{~mm} \times 4 \mathrm{~mm} \times 4 \mathrm{~mm}$ for each. Signals are readout from Multi-Pixel Photon Counter (MPPC) from both sides of each bar to determine the 1-dimensional position of bar. This PSA is utilized in elastic scattering for neutrons, and Compton scattering in gamma-rays. The 2 nd part is $12 \times 12$ GAGG(Ce) scintillator array (GSA) with $6 \mathrm{~mm}$ cubic for each crystal, readout by MPPCs. This GSA detects gamma-rays in photo-absorption, also can detect thermal neutrons due to large cross-section of neutron capture for Gd. The third one is anti-coincidence detector which consists oi 6 plastic scintillators which cover the whole PSA to reject charged particles. In this configuration, our detector is sensitive to both $30-100 \mathrm{MeV}$ neutrons 
and 100-3000 keV gamma-rays. In principle, it can also localize both neutrons and gamma-rays using two elastic scattering events and Compton scattering+photo-absorption events, respectively. An example of tracks for $100 \mathrm{MeV}$ neutron and $1 \mathrm{MeV}$ gamma-ray is shown if Figure 2. The results are obtained from Geant-4 simulation [11].
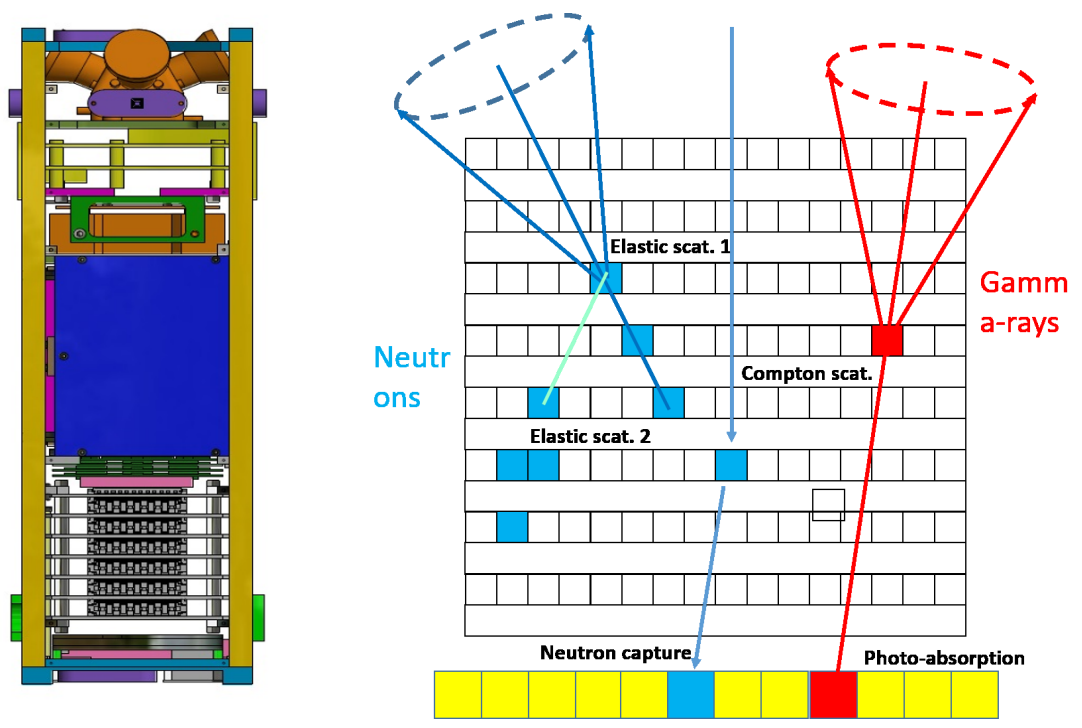

Figure 1: (Left) 3U CubeSat Configuration (Solar paddles are removed). The neutron/gamma-ray detector is put at the center of the sallite as shown by blue. (Right) Detector configuration, consisting of plastic scntillator and GAGG(Ce) scintillator array. Neutrons can be detected by elastic scattering (shown by cyan), while gamma-rays are detected by Compton scattering + photo-absorption (by red).

Table 2: Characteristics of the neutron/gamma-ray sensor.

\begin{tabular}{cc}
\hline \hline Items & Values \\
\hline Detector Size & $10 \times 10 \times 12 \mathrm{~cm}$ \\
Weight $(\mathrm{kg})$ & 2 \\
Power $(\mathrm{W})$ & 4 \\
Energy & $30-100 \mathrm{MeV}$ (neutrons), $100 \mathrm{keV}-2 \mathrm{MeV}$ (gamma-rays) \\
Plastic Scinti. & $64 \mathrm{~mm}$ cubic $(256 \mathrm{pcs}), 4 \times 4 \times 64 \mathrm{~mm}$ for each \\
GAGG(Ce) & $12 \times 12$ array, $6 \mathrm{~mm}$ cubic for each \\
Photo-sensor & MPPC $3 \times 3 \mathrm{~mm}^{2}$ (plastic) \\
& $4 \times 4$ array with $6 \times 6 \mathrm{~mm}^{2}$ each (GAGG) \\
\hline
\end{tabular}

\section{Results from Bread Board Model (BBM)}

To confirm the sensor performance and establish ASIC readout system, we have constructed Bread Board Model(BBM) of sensors and electronics. The BBM consists of plastic scintillator array (PSA) with 4 layers (left panel of Figure 3) and ond GAGG scintillator array (GSA) with $4 \times 4$ array (left panel of Figure 5), and processing board with 4 Application Specified Integrated 

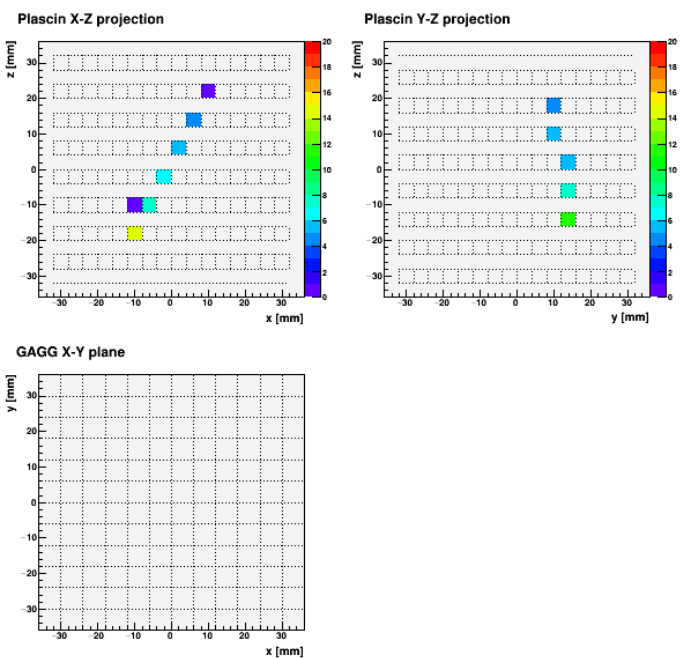
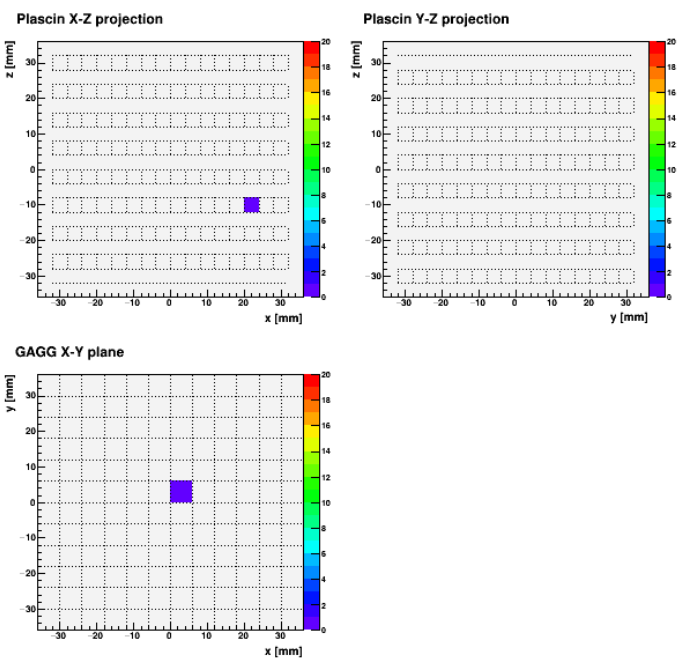

Figure 2: Geant-4 simulation results for an example of Neutron $100 \mathrm{MeV}(\mathrm{Left})$ and Gamma-ray 1 $\mathrm{MeV}$ (Right). The upper two panel indicate $\mathrm{X}-\mathrm{Z}$ and $\mathrm{Y}-\mathrm{Z}$ plane of plastic scintillator array, and lower panel indicate GAGG(Ce) $12 \times 12$ array.

Circuits(ASICs) and one Field Programmable Gate Array (FPGA(. The bare chip of IDE3380 provided by IDEAS is packaged in a TQFP package with a size of $12 \mathrm{~cm} \times 12 \mathrm{~cm}$.

First we have irradiate collimated gamma-rays to plastic scintillator bars to confirm 1-dimensional (1D) position sensitivity of bars. The bar surface is roughened, and wrapped with thin Aluminum Mylar. The 1D position along the bar is estimated from ratio of light outputs for each side of the MPPCs, and its measure is $\log \left(E_{1} / E_{2}\right)$ where the $E_{1} / E_{2}$ means light output ratio. Right panel of Figure 3 shows relation between real position and its position measure, showing clear linear correlation. The position resolution is found to be $2-3 \mathrm{~mm}$ at $1 \sigma$ for $662 \mathrm{keV}$ Compton scattering events below $478 \mathrm{keV}$. We also investigated tracking performance of cosmic-ray muons, and energy deposits in bars follows the Landau distribution. The $478 \mathrm{keV}$ Compton edge of $662 \mathrm{keV}$ gammarays is almost consistent with values estimated from peak of Landau distribution. To confirm the energy resolution for protons, We have a plan of proton-irradiation beam tests for this array this fall.

We have also irradiated gamma-ray radiation source to $\operatorname{GAGG}(\mathrm{Ce}) 4 \times 4$ array. The array is composed of 16 polished crystals with $6 \mathrm{~mm}$ cubic, which are attached by glues including white reflector based on $\mathrm{BaSO}_{4}$. The crystal size and location are matched to the MPPC $4 \times 4$ array. When we take 16 channel data with ASIC, we found that there were two effects: Saturation in MPPC pixels due to large light yield of GAGG(Ce) crystals and light leakage among adjacent pixels due to incompleteness of individual pixel separation. The saturation effect is corrected by $1-\exp \left(-Q / Q_{\max }\right)$ where $Q$ is the charge and $Q_{\max }$ is the maximum charge determied by pixel number and scintillator decay time. The light leakage is corrected by a linear function between two pixels $(i, j$ where $i, j=1,2, . .16)$ for all 256 combinations. The resultant pixel correction is shown in Figure 4. After the light leak correction, we can judge how many pixels are reacted clearly. The Full-Width-Half-Maximum (FWHM) energy resolution for corrected one is about $6 \%$ at $662 \mathrm{keV}$ at 20 degree $\mathrm{C}$ (right panel of Figure 5), and is inversely proportional to square root of produced charge $(\propto 1 / \sqrt{Q})$ expected from statistical distribution of photo-electrons. This method can be 


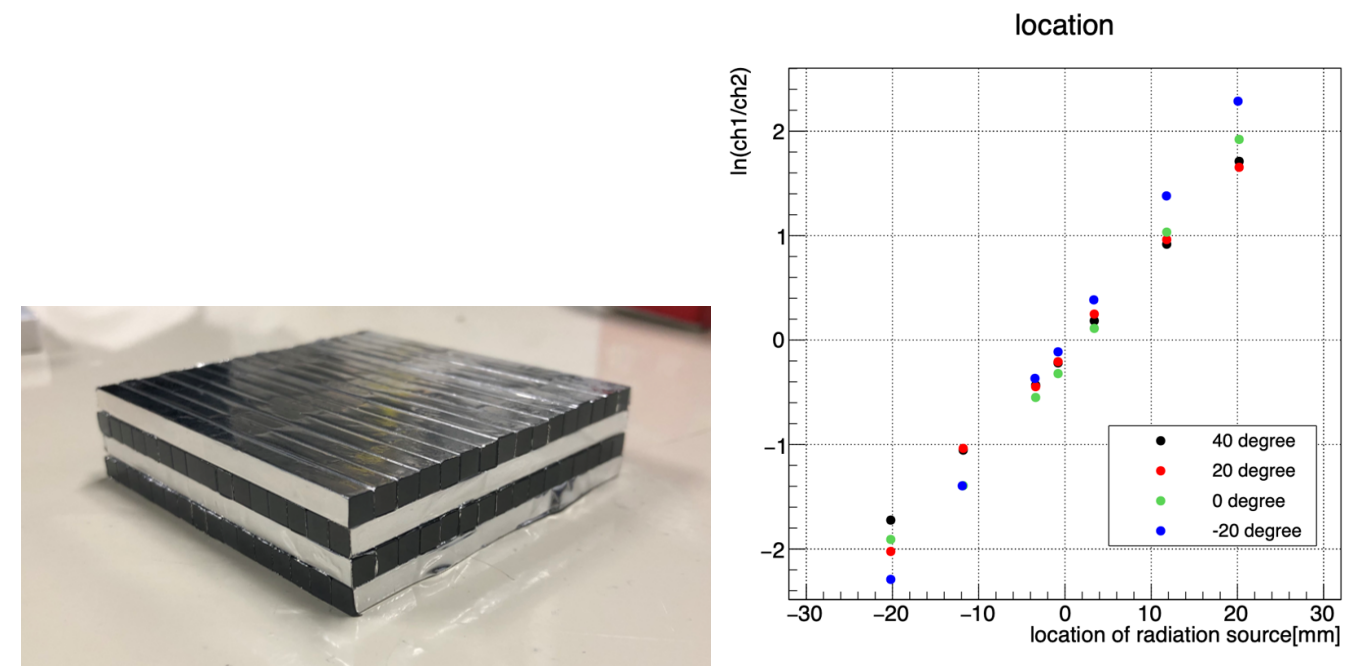

Figure 3: (Left) Bread Board Model(BBM) of Plastic Scntillator Array with 16 bars $\times 4$ layers. (Right) Relation between real position of radiation source ${ }^{137} \mathrm{Cs}$ and position measure $\log (\mathrm{E} 1 / \mathrm{E} 2)$ where E1 and E2 are light outputs from each side of one bar.

applied for $12 \times 12$ array of the flight model. The performance of Compton camera combined plastic and GAGG array is still under way.
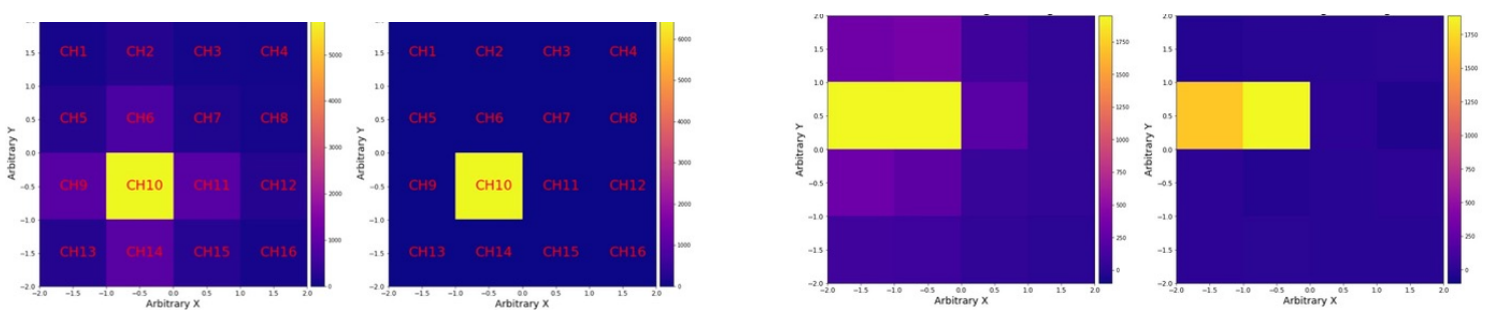

Figure 4: (Left) Pixel distribution of sigle hit event in GAGG(Ce) $4 \times 4$ array. (Right)Two-hit events. Each left figure indicates uncorrected light-leakage distribution, while right figure indicates corrected one. The weak light leakage from bright pixels is diminished in right figure.

\section{Conclusion}

In order to realize high sensitive solar neutron observations, we are designing and developing a 3U CubeSat SONGS planned for launch around 2024 at the next solar maximum. This is the first CubeSat mission dedicated for solar neutron observations. The novel neutron/gamma-ray detector onboard SONGS consists of plastic and GAGG(Ce) scntillator array readout by Silicon photo-multipliers, and achieve very compact size, low power consumption, and light weight. We are currently evaluating performance of breadboard model(BBM), and will construct flight model in 2023. We are also considering to apply it to other motivation such as search for water resource on the moon and neutron lifetime measurement in space. 

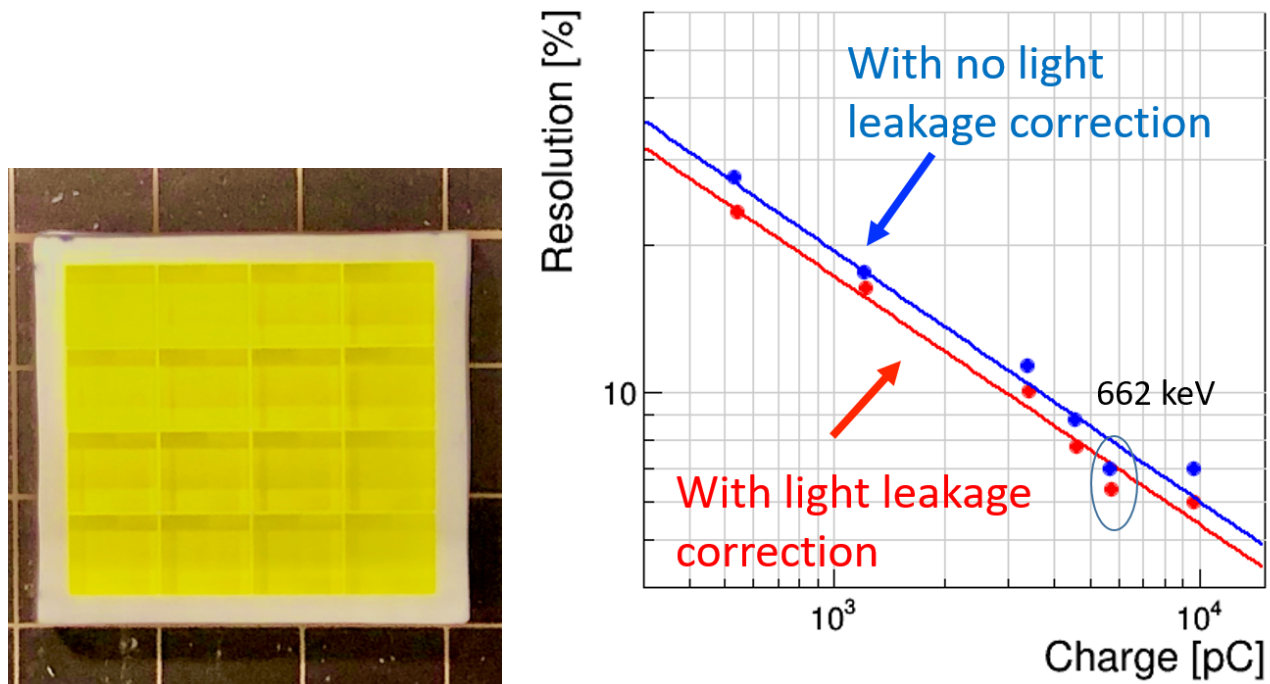

Figure 5: (Left) GAGG(Ce) Scntillator Array. (Right) Gamma-ray energy resolution as a function of charge. The FWHM energy resolution at $662 \mathrm{keV}$ is about $6 \%$ for 20 degree C.

\section{Acknowledgments}

This research has been financially supported by Toyoaki Scholarship Foundation and JSPS Grant-in-Aid for Scientific Research (KAKENHI) Grant Number JP18H03700 (KY).

\section{References}

[1] Parker, E. N.: The Solar-Flare Phenomenon and the Theory of Reconnection and Annihiliation of MagneticFields, Astrophysical Journal Supplement, 8 (1963), pp. 177.

[2] Ajello, M., Baldini, L., Bellazzim, B., et al.: First Fermi-LAT Solar flare catalog, Astrophysical Journal Supplement, 252 (2021), pp. 13.

[3] Matsubara, Y., Muraki, Y., Masuda, K, et al.: Observation of Solar Neutrons by the WorldWide Network ofSolar Neutron Detectors, Proceedings of the 26th International Cosmic Ray Conference, (1999), 42

[4] Yu, X. X., Lu, H., Chen, G. T., Li, X. O., Shi, J. K., Tan, C. M.: Detection of solar neutron events and their theoretical approach, New Astronomy, 39 (2015), pp. 25-35

[5] Chupp, E. L. Forrest, D. J., Ryan, J. M., et al.: A Direct Observation of Solar Neutrons Following the 0118 UTFlare on 1980 June 21, Astrophysical Journal, 263 (1982), pp. L95L98.

[6] Muraki, Y., Koga, K., Goka, T., Matsumoto, H., Obara, T., Okudaira, O., Shibata, S., Yamamoto, T.: Measurement by FIB on the ISS: Two Emissions of Solar Neutrons Detected?, Advances in Astronomy, (2012), article ID 379304. 
[7] Koga, K., Muraki, Y., Masuda, S., Shibata, S., Matsumoto, H.; Kawano, H., Measurement of Solar Neutronson 05 March 2012, Using a Fiber-Type Neutron Monitor Onboard the Attached Payload to the ISS, SolaPhysics, 292(8) (2017), pp. 115-130.

[8] Lawrence, D. J., Feldman, W. C., Goldsten, J. O., Peplowski, P. N., Rodgers, D. J., Solomon, S. C., Detection and characerization of $0.5-8 \mathrm{MeV}$ neutrons near Mercury: Evidence for a solar origin, Journal of Geophysical Research: Space Physics, 119 (2014), pp. 5150-5171.

[9] Yamaoka, K., Babazaki, Y., Hayashi, Y., et al.: Solar Neutron and Gamma-ray Monitor on the ChubuSat-2 Satellite, Transaction of the Japan Society for Aeronautical and Space Sciences, AerospaceTechnology Japan 14(ists30), (2016), Pf_141-146.

[10] Yamaoka, K., Tajima, H., Miyata, K, et al.: Solar Neutron Spectrometer Onboard a 3U CubeSat, Transaction of the Japan Society for Aeronautical and Space Sciences, Aerospace Technology Japan, 19(3) (2021), pp. 354-359.

[11] Agostinelli, S., Allison, S., Amako, K., et al.: Geant4 simulation toolkit Nuclear Instrumens tMethods in Physical Research Section A, 506 (3) (2003), pp. 250-303.

[12] Nobashi, D., Yamaoka, K., Tajima, H., Ito, K., Performance evaluation of GAGG(Ce)/LFS scintillator + MPPC array readout with ASIC, Nuclear Instrumens tMethods in Physical Research Section A, 986 (2021), 164811. 